\title{
A Vehicle Behavioral Analysis of the Signal Pre-warning Information Provided to the Driver
}

\author{
Toshiyuki Nakamura ${ }^{1}$, Tatsuki Nakayama ${ }^{1}$, Nobuhiro Uno ${ }^{1}$ and Keiichi Yamamura ${ }^{2}$ \\ 1. Graduate School of Engineering, Kyoto University, Kyoto 615-8540, Japan \\ 2. Nihonkai Consultant Co., Ltd., Kanazawa 921-8042, Japan
}

\begin{abstract}
In this study, we analyze whether there is a change in driver actions because of voice navigation information provided by utilizing ITS (intelligent transport systems). Specifically, when a vehicle approaches a signalized intersection, the driver is provided with the voice navigation information that the signal light will shortly change red. We focus on what is effective when the voice navigation information is provided. Even if the timing of the provision of voice information is delayed, we have evaluated whether the vehicle is able to stop safely. From the results of the analysis, by providing voice navigation information, we know that a vehicle will stop safely at a signalized intersection. Even if the information provided by voice navigation has been delayed, it could be shown to be safe compared with the case where no information was provided.
\end{abstract}

Key words: Driving simulator, signal pre-warning information, vehicle behaviour.

\section{Introduction}

Due to the progress of ITS (intelligent transport systems), there is no doubt that our lives will be more comfortable and safer. At present, there are a variety of ITS-related supports for when we drive a vehicle. For example, there is auto cruise control, which is useful when we are driving on highways, and a brake system for collision damage mitigation in which the driver's vehicle can sense the vehicle in front and other obstacles. Recently, automated vehicle operation technology has attracted attention.

About 629,000 traffic accidents occurred in Japan in 2013. Traffic accidents produce a large social and personal loss. It is necessary to reduce the number of traffic accidents. Under such circumstances, ITS has contributed to the reduction of traffic accidents.

This study aims to analyze whether there is a change in the driver's actions because of the voice navigation information provided by utilizing ITS. Specifically, when the vehicle approaches a signalized

Corresponding author: Toshiyuki Nakamura, Ph.D., assistant professor, research fields: travel behavior analysis, traffic engineering and transportation planning. intersection, the driver is provided with the voice navigation information showing that the signal light will shortly change red. We focus on what is effective in the case where the voice navigation information is provided. Even if the timing of the provision of voice information is delayed, we have evaluated whether the vehicle is able to stop safely.

It is realistically difficult to collect the data on a real road as it is possible that this will lead to traffic accidents. The data collection in this study uses a driving simulator.

In a similar study to this research, Lee et al. [1] tried evaluating RECASs (rear-end collision avoidance systems) using driving simulators. The results show that early warnings helped drivers to avoid collisions more than late warnings or no warnings. Some studies [2-6] analyzed the effect of alarm timing on driver response to alarm malfunctions from the perspective of driver trust. As a result, drivers who experience late alarms are reluctant to respond to a false alarm and are not influenced by a missing alarm. However, drivers who experience early alarms tend to respond to false alarms and suffer a 
delayed response to critical situations following missing alarms. Yamada and Kuhar [7] investigated the behavioral and safety effects of driver dependence on such a warning system, especially when the warning system is unreliable. The result of the experiment was that drivers are affected by the accuracy of the warning system. The mean driving speed decreased as the missed detection rate increased, demonstrating a decrease in the driver's reliance on warnings when the system was less effective in detecting threats. It is clear that the provision of information usually influences driving behaviour. This study focuses on communicating with the signal and the vehicle.

\section{Overview of Driving Simulator Experiment}

It is realistically difficult to collect data from real roads, and it is possible that doing so will lead to traffic accidents. Therefore, the data collection in this study uses a driving simulator. We selected a driving simulator experiment targeting a national road, which is a general road, such as National Route 1 . The experimental target road has two lanes, signalized intersections and a speed limit of $60 \mathrm{~km} / \mathrm{h}$. The driver repeatedly uses the same road and intersection. The driving simulator experiment is performed to provide information on the navigation system using a voice. In the experiment, when the vehicle approaches the intersection, the signal light changes to red. The driver is provided with voice information through the navigation system. The voice states that, "the signal will shortly turn red, please be aware of a rear-end collision".

We undertook experiments on three different types of method. One is that the information is provided at the right time. The second is that the information is provided with a time lag. The information with a time lag refers to information provided in closer proximity to the signalized intersection. The third is that no information is provided. We assumed that the results of the experiments would show that the driver who is provided with information will be safe, compared when there is no information, even if the driver is provided with information with a time lag. We are experimenting with the idea that when the driver approaches the intersection, we combine the case where the signal does turn to not only yellow and red, but also blue or the beginning of the red pattern. The number of drivers is 34 , and all of them drive vehicles on a daily basis (drivers in their $20 \mathrm{~s}: 18$ people; $30 \mathrm{~s}$ : 6 people; 40 s: 6 people; 50 s: 4 people). Before starting the experiment, the driver has sufficient practice time to get used to a driving simulator.

\section{Setting of Analysis Section and Analysis Indices}

Vehicle behavior data from DS (driving simulator) referred to as log data is collected for each of about $0.003 \sim 0.05 \mathrm{~s}$, as well as the vehicle location, acceleration velocity, vehicle speed at the time and so on. In consideration of the road structure, the signalized intersection, we define three sections (four intervals): the interval of non-provision of information, the interval of provision of information and the interval of the switching of the signal phase (Fig. 1).

We defined the start point where the information provided by voice navigation is made, that is, where the signal light is switched to red from yellow. The provision of information interval is divided into the first half and the second half, because of this timing to start the voice navigation.

Analysis indices are used, four of which will be described below.

\subsection{Travel Speed}

The traveling speeds of all drivers are aggregated by every meter and are calculated by taking an average value. We have shown the change by distance from the stop line. To determine what the difference is for the average travel speed in each interval, we perform a statistical test. The interval average travel 


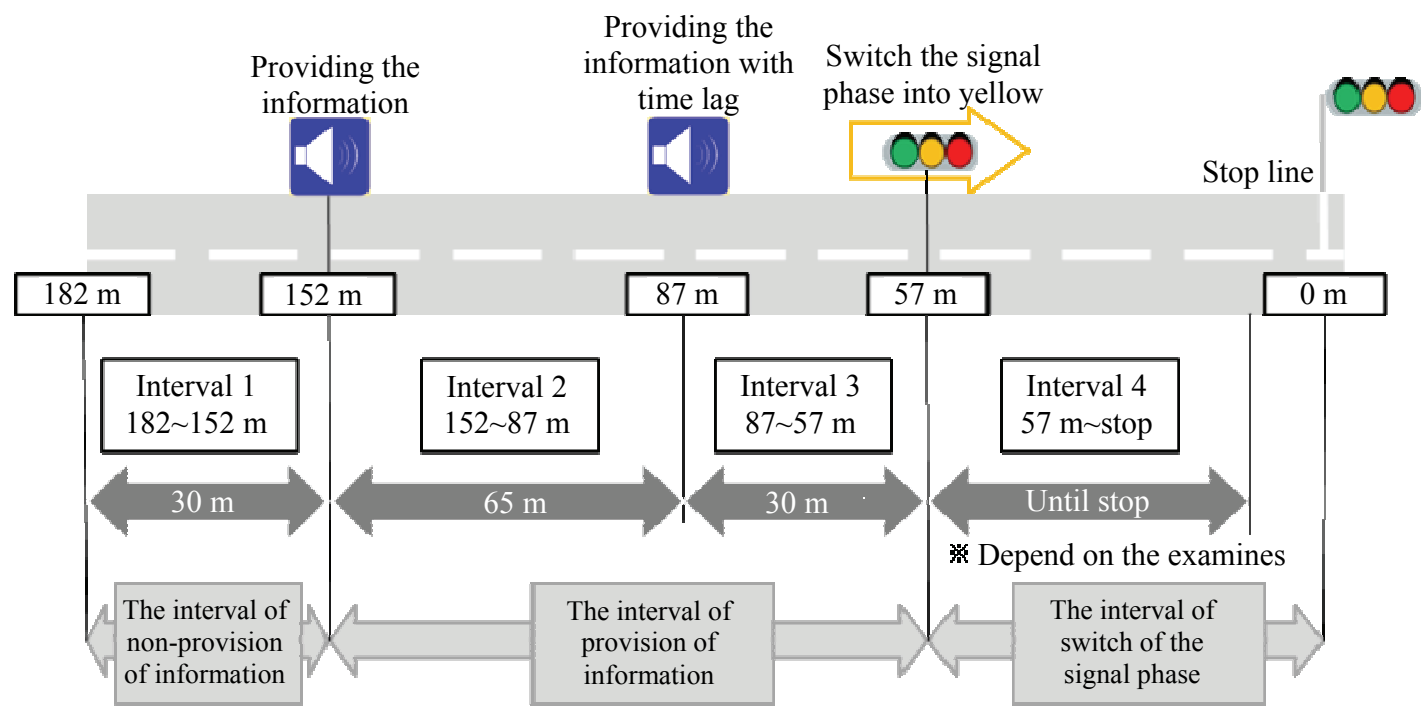

Fig. 1 Situation of analysis section.

speed is defined as the value obtained by dividing the interval length in the interval travel time.

\subsection{Brake Pedal Depression and Accelerator Pedal Depression}

The degree of depression of the brake/accelerator for data collection is: no depression of the brake pedal $=0$, and continuous depression to the maximum $=1$. In this study, we analyze the depression degree of the brake and accelerator at a certain distance from the stop line.

\subsection{Maximum Deceleration}

Deceleration is defined as a value obtained by dividing the decrease of the speed of the vehicle behavior data between the last two points in time increments. We use the maximum value within the interval as the average value of maximum deceleration. We think that the maximum deceleration is the evaluation index that can evaluate the operating conditions of the previous stop at the intersection. If the maximum deceleration is large, the driver will stop during sudden braking. And, we analyze the position where the maximum deceleration occurred.

\subsection{Minimum PICUD of Average Value}

We analyze the gap acceptance and potential risk of collisions. The potential risk of a collision utilizes PICUD (possibility index for collision with urgent deceleration) [8]. Of course, due to the gap acceptance and potential risk of collisions, there are some indexes. For example, Brown et al. [9] proposes the kinematics-based and perceptual-based rear-end collision avoidance algorithms over a range of collision situations as a simple deterministic model of driver performance.

PICUD adapted to this study is an index to evaluate the possibility that two consecutive vehicles might collide, assuming that the leading vehicle applies its emergency brake. PICUD is a conflict indicator that uses the relative distance and speed, deceleration and the reaction delay time calculated by the following vehicle and the preceding vehicle. If PICUD takes a smaller value than zero, the preceding vehicle suddenly stops and both vehicles crash. PICUD is calculated by Eq. (1):

$$
P I C U D=\frac{V_{1}^{2}}{-2 a_{1}}+s_{0}-\left(V_{2} \Delta t+\frac{V_{2}^{2}}{-2 a_{2}}\right)
$$

where:

$V_{1}, V_{2}$ represent the velocity of the preceding and following vehicles, respectively, when the preceding vehicle applies an emergency brake;

$s_{0}$ indicates the distance between the two vehicles at 
the point in time when the preceding vehicle applies the emergency brake;

$\Delta t$ indicates the reaction time of the driver of the following vehicle;

$a$ represents the deceleration rate of both the preceding and following vehicles.

\section{Results of Vehicle Behavioral Analysis}

\subsection{Result of Travel Speed}

The change in the average value of the traveling speed of all drivers obtained in 1-m intervals by the distance from the stop line is shown in the upper left side in Fig. 2. From the graph of this distribution, when the driver is provided with the information with better timing, the result shows that they tend to reduce the travel speed from Interval 2. In the case of providing information with a time lag, they tend to reduce the traveling speed from Interval 3.

If there is no information, they suddenly tend to reduce the traveling speed in Interval 4. The upper right side in Fig. 2 is the result of a $t$-test among intervals by the average values of no information, providing information and providing information with a time lag. The values on the lines show the $t$-value, and the thickness of the line shows the level of significance. The results of the analysis show no significance in Interval 1 among three types, but the providing information and the other two types have a difference in Interval 2. Further, the trend has become more prominent in Intervals 3 and 4.

\subsection{Result of Brake and Acceleration Pedal Depression}

The middle left side (Fig. 2) shows the distribution of the average degree of depression of the brake for all drivers. As mentioned, the maximum braking pedal depression in Interval 4 has a large value when there is no information for the driver. The trend is increased to the order ("no information" > "providing information with time lag" > "providing information"). The results of the level of significance have been obtained from the difference between the $t$-test. In other words, when providing the information signals to replace the red from the yellow, the effect of suppressing sudden braking is suggested. The lower left side (Fig. 2) shows a distribution of the average value of the accelerator depression degree of all drivers. As mentioned, it can be seen that the point at which the accelerator intensity decreases and the start point where the information provided has been offered are almost the same. In addition, in Interval 4, regarding no information, there is a tendency that the driver steps on the accelerator just before it turns red from yellow. It can be seen that it is already taking values close to zero by the providing information. As the results of the $t$-test show, in Intervals 2 and 3 , all patterns are seen to be statistically significant.

\subsection{Maximum Deceleration}

The maximum value of the deceleration in Interval 4 is shown in Fig. 3. Fig. 3 contains no information, providing information with time lag and providing information. The vertical line of Fig. 3 represents the number of samples (drivers), and the horizontal line represents the distance from the intersection. It seems that greater maximum deceleration occurs at a position near the intersection when there is no information. If there is providing information, deceleration does not occur so much near the intersection; Further, it is seen that the degree of deceleration in excess of $5 \mathrm{~m} / \mathrm{s}^{2}$ is the lowest of the three types. On the other hand, if there is the providing information with time, the distribution of the maximum deceleration varies widely. In other words, regarding the maximum value and its occurrence position of deceleration, it is considered that personal difference tends to be larger. As compared with the case where there is no information, large deceleration at the nearby intersection has decreased. When the appropriate information is provided to the driver, a large deceleration at the nearby intersection has not occurred. As can be seen 
(a) Travel speed
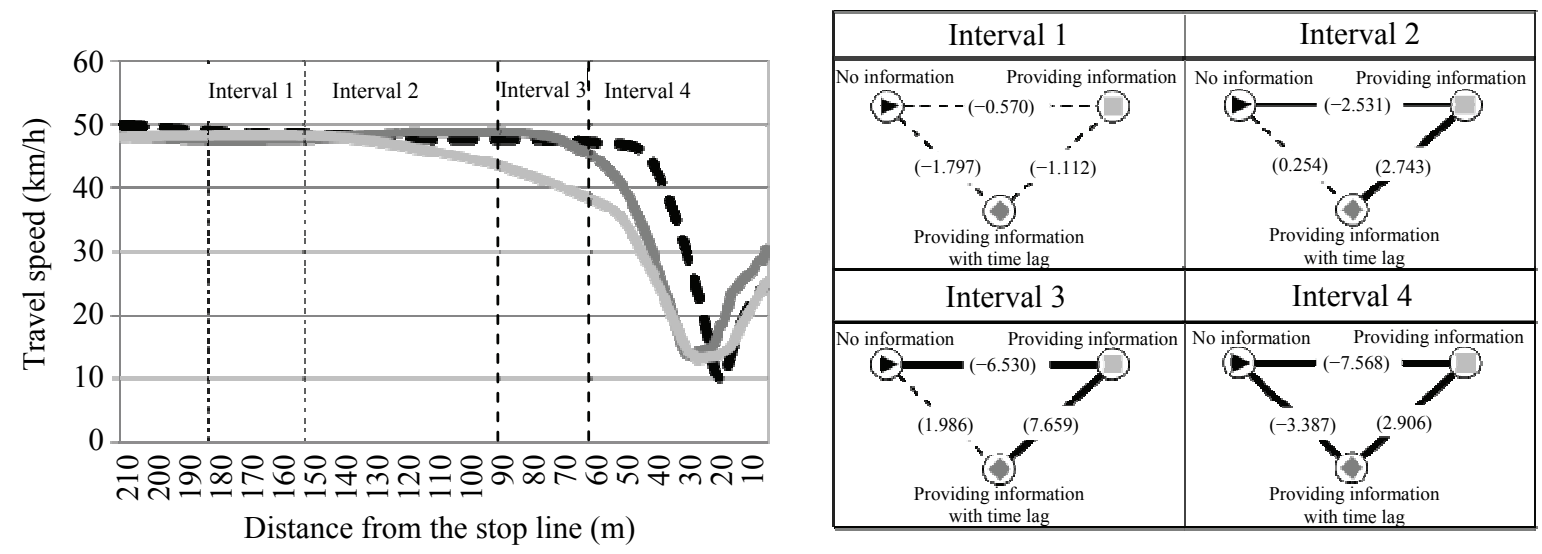

(b) Brake pedal depression
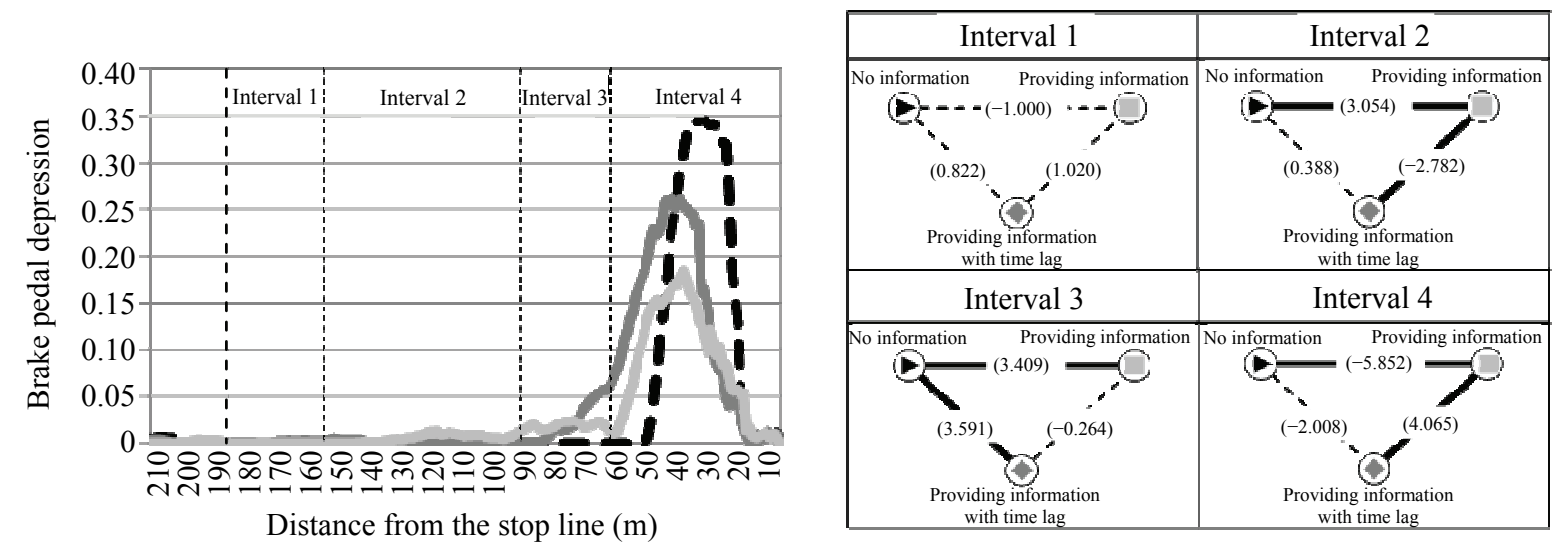

(c) Acceleration pedal depression
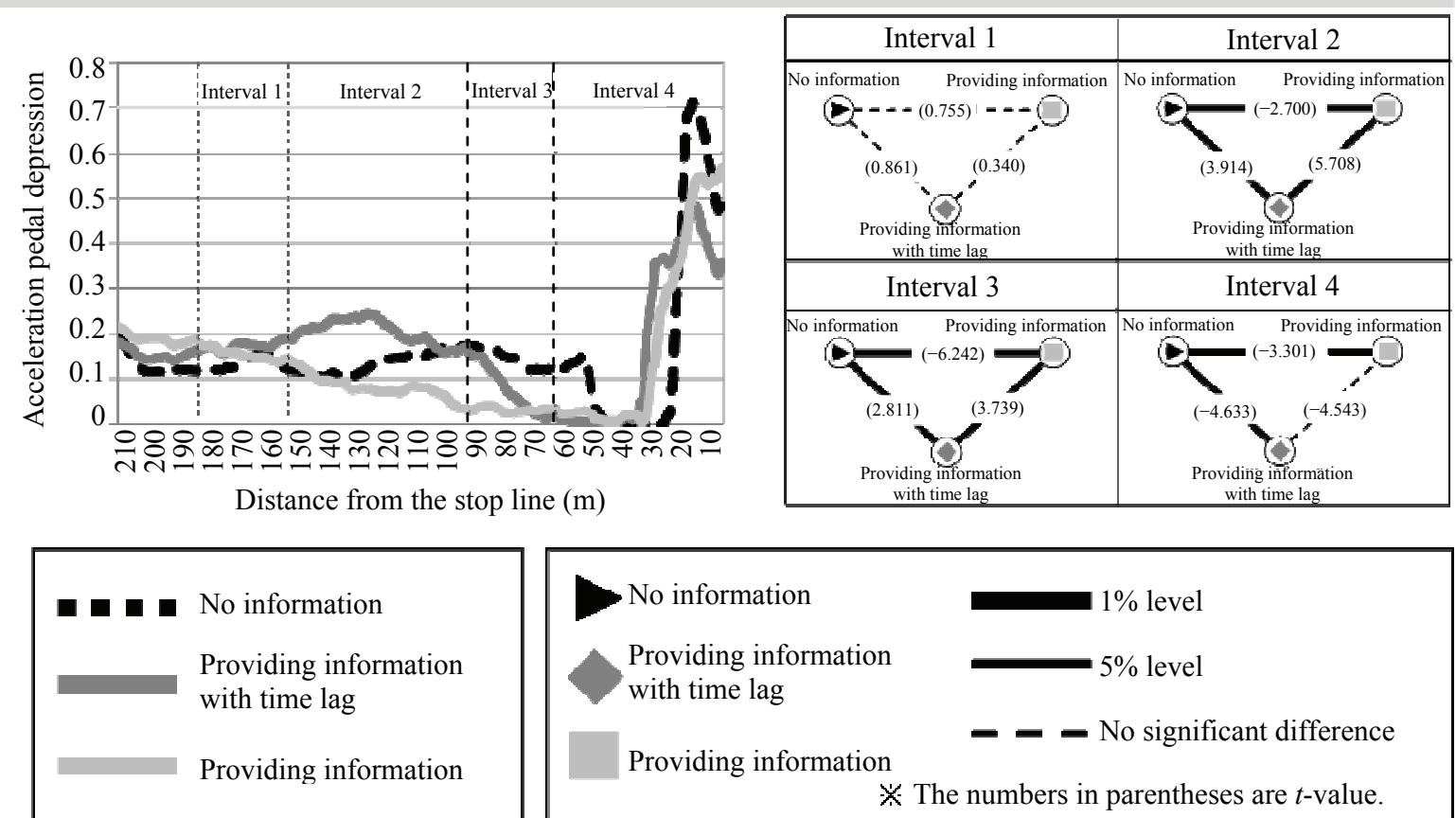

Fig. 2 Results of: (a) travel speed; (b) brake pedal depression; (c) acceleration pedal depression. 
No information

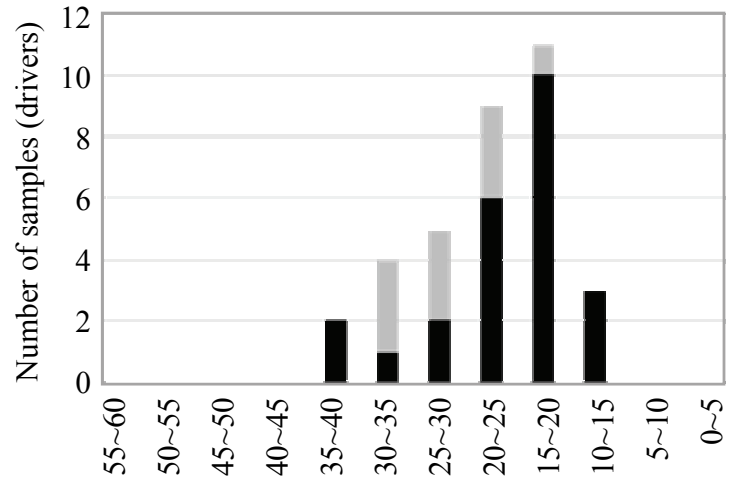

Distance from the stop line $(\mathrm{m})$

Providing information

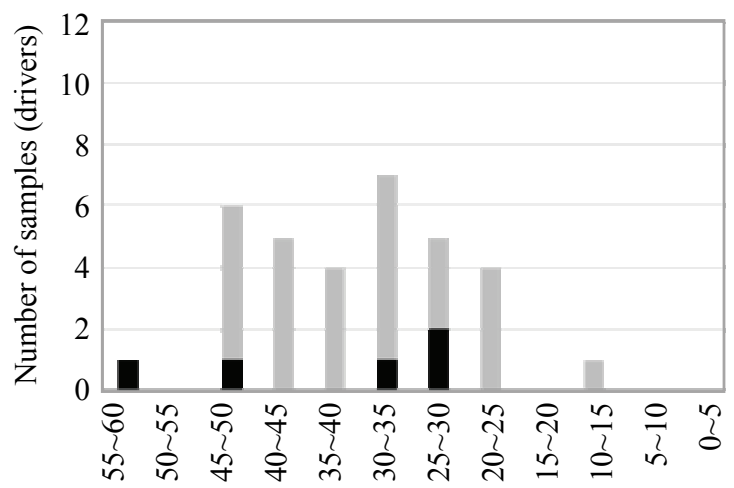

Distance from the stop line $(\mathrm{m})$
Providing information with time lag

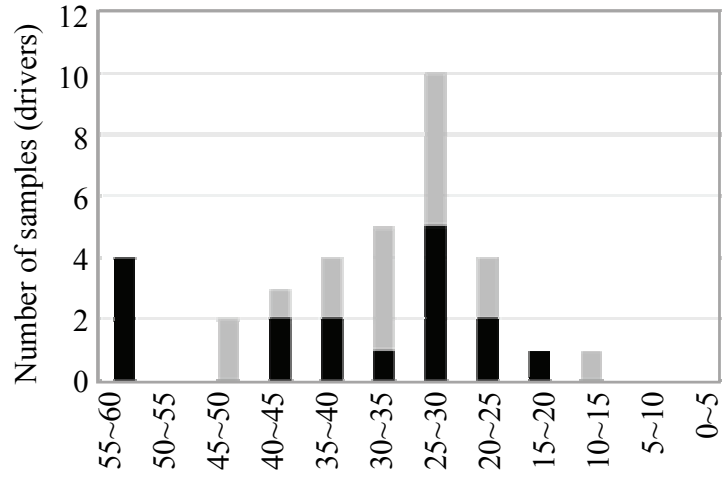

Distance from the stop line $(\mathrm{m})$

Maximum deceleration is

more than $5 \mathrm{~m} / \mathrm{s}^{2}$

Maximum deceleration is

less than $5 \mathrm{~m} / \mathrm{s}^{2}$

Fig. 3 Maximum value of deceleration in Interval 4.

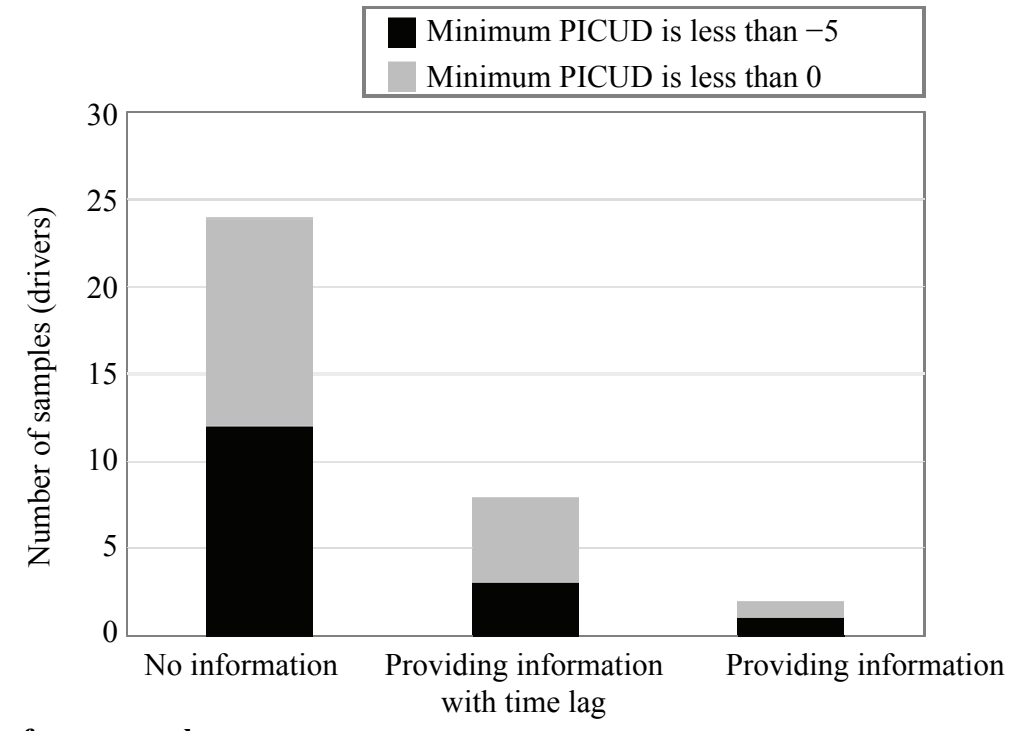

Fig. 4 Minimum PICUD of average value. 
from these results, by providing information to the driver, even when it has a time lag, it can be confirmed that there is a decrease in rapid deceleration near the intersection.

\subsection{Minimum PICUD of Average Value}

When it is described again, if PICUD has a smaller value than 0 , the preceding vehicle suddenly stops and both vehicles crash. It shows the minimum PICUD of the average value in Fig. 4. According to this result, when it was performed to provide information, the ratio of the minimum PICUD which is less than 0 is seen to clearly be lower. Even if information with a time lag is provided, it seems that the risk of collision is lower than with no information. Furthermore, if the minimum PICUD is less than -5 , this tendency is remarkable.

\section{Conclusions}

In this study, we analyzed the effect of ITS technology for reducing traffic accidents in the future using driving behavior. As shown in the results, the information provided by voice navigation reduces rear-end traffic accidents. If the information was provided with a time lag, it was possible to reduce rear-end traffic accidents more than when no information was delivered. However, there is a problem with the introduction of these technologies. For example, regarding such a system on a real road, we assume that when it is providing information to forward signals replace the red to driver, all drivers really provided to stop, rather drivers previously known that replaces the red are accelerated in order to enter the intersection. Thus, considering the problem in operation, technology in this study has to be suitable for real society. We recognize that ITS is not a panacea, and that we must also consider the use of other methods in the future. As a result of this study, we hope that ITS technology will help reduce traffic accidents.

\section{References}

[1] Lee, J. D., McGehee, D. V., Brown, T. L., and Reyes, M. L. 2002. "Collision Warning Timing, Driver Distraction, and Driver Response to Imminent Rear-End Collisions in a High-Fidelity Driving Simulator." Human Factors: The Journal of the Human Factors and Ergonomics Society 44 (2): 314-34.

[2] Genya, A., and Richardson, J. 2004. "The Human Factors of Collision Warning Systems: System Performance, Alarm Timing, and Driver Trust." In Proceedings of the Human Factors and Ergonomics Society Annual Meeting, SAGE Publications, 2232-6.

[3] Gupta, N., Bisantz, A. M., and Singh, T. 2002. "The Effects of Adverse Condition Warning System Characteristics on Driver Performance: An Investigation of Alarm Signal Type and Threshold Level." Behaviour and Information Technology 21 (4): 235-48.

[4] Bliss, J. P., Gilson, R. D., and Deaton, J. E. 1995. "Human Probability Matching Behaviour in Response to Alarms of Varying Reliability." Ergonomics 38 (11): 2300-12.

[5] Meyer, J., and Bitan, Y. 2002. "Why Better Operators Receive Worse Warnings." Human Factors 44 (3): 343-53.

[6] Maltz, M., Sun, H., Wu, Q., and Mourant, R. 2004. "Use of an In-vehicle Alerting System for Older and Younger Drivers: Does Experience Count?." Transportation Research Record 1899: 64-70.

[7] Yamada, K., and Kuchar, J. K. 2006. "Preliminary Study of Behavioral and Safety Effects of Driver Dependence on a Warning System in a Driving Simulator." IEEE Transactions on Systems, Man and Cybernetics, Part A: Systems and Humans 36 (3): 602-10.

[8] Uno, N., Iida, Y., Yasuhara, S., and Itsubo, S. 2003. "A Microscopic Analysis of Vehicular Behavior at Weaving section Considering Traffic Conflicts." Presented at 10th World Congress on Intelligent Transportation Systems, Madrid, Spain.

[9] Brown, T. L., Lee, J. D., and McGehee, D. V. 2001. "Human Performance Models and Rear-End Collision Avoidance Algorithms." Hum. Factors 43 (3): 462-82. 